\title{
Breast reconstruction: current and future options
}

This article was published in the following Dove Press journal:

Breast Cancer:Targets and Therapy

16 August 2011

Number of times this article has been viewed

\author{
Henry Paul Jr' \\ Tahira I Prendergast ${ }^{2}$ \\ Bryson Nicholson ${ }^{2}$ \\ Shenita White ${ }^{2}$ \\ Wayne Al Frederick 2,3 \\ 'Departments of Plastic Surgery, \\ ${ }^{2}$ General Surgery, Howard University \\ Hospital, ${ }^{3}$ Cancer Center, Howard \\ University, Washington, DC, USA
}

Correspondence: Henry Paul Howard University College of Medicine, 204 I Georgia Ave, NW, Tower Suite 4100-B, Washington, DC 20060, USA

Tel + I 202865 I286

Fax + I 2028653063

Email hpaul@howard.edu

\begin{abstract}
When initiated by the devastating diagnosis of cancer, post ablative breast restoration has at its core the goal of restoring anatomic normalcy. The concepts of body image, wholeness, and overall well-being have been introduced to explain the paramount psychological influence the breast has on both individuals and society as a whole. Hence, a growing subspecialty has been established to recreate or simulate the lost breast. At least one third of breast cancer victims consider breast reconstruction. Breast reconstruction post mastectomy may be offered at the time of mastectomy or delayed post mastectomy after adjuvant therapy. This may be utilizing autologous tissues or implants and each has risks and benefits, especially when considering adjuvant therapy. In addition, there has been a move away from a traditional mastectomy to less invasive, but still curative procedures, such as skin-sparing and nipple-sparing mastectomy. These procedures provide the breast envelope to facilitate reconstruction. This paper reviews the primary issues in breast reconstruction, as well as their psychologic, oncologic, and social impact.
\end{abstract}

Keywords: breast restoration, body image, breast reconstruction, mastectomy

\section{History of breast reconstruction}

Breast cancer is the second leading cause of cancer-related death among females in the US, and affects one in eight women in their lifetime. ${ }^{1,2}$ Advances in medical research have dramatically improved the treatment of this disease over the last 50 years. In addition to chemotherapy and radiation therapy, partial and complete mastectomy of diseased and contralateral breasts has become more frequent. However, these surgical modalities often leave patients with anatomical deformities and altered body image, along with the psychologic impact. ${ }^{3,4}$

Breast reconstruction is a dynamic and multidisciplinary approach that has grown into a solution for many breast cancer patients treated with surgery. Breast reconstruction began as a means to reduce chest wall complications and deformities from mastectomy, with the goal to recreate symmetry and contour while avoiding delay in adjuvant cancer treatment.

Breast reconstruction was introduced over a century ago as a surgical modality for rebuilding the anatomic breast using autologous tissues and prosthetic implants. ${ }^{2,5}$ Dating back to the late 1800 s, procedures performed to reconstruct the breast included the introduction of foreign material and the transfer of healthy (and at times pathologic) autogenous tissues. Beginning in the late 19th century, physicians used techniques such as transferring healthy breast tissue on a pedicle to reconstruct the contralateral breast. ${ }^{5}$ 
The 20th century ushered in the use of flaps from the pectoralis and latissimus dorsi muscles for cosmetic improvement. ${ }^{5}$ In the late 1900s, musculocutaneous flaps were used and donor sites expanded beyond the pectoralis and latissimus dorsi muscles to include tissues from other areas, such as the abdominal wall, buttock, flank, and thigh. ${ }^{5}$ This expansion of autologous tissue use has allowed for the development of a wide array of surgical options, ie, transverse rectus abdominis myocutaneous (TRAM) flap, superficial inferior epigastric artery (SIEA) perforator flap, deep inferior epigastric perforator (DIEP) flap, thoracodorsal artery perforator flap, and others. ${ }^{6}$

In addition to physicians using the body's tissues to reconstruct the anatomy, prosthetic alloplastic materials were implanted or injected to alter or restore the shape and size of the breast. ${ }^{5}$

The silicone breast implant was introduced in 1962 and has steadily evolved since. ${ }^{5}$ Cronin and Gerow experimented with implantation of silicone shells filled with either dextran or electrolyte solution in dogs for days to months without complications. ${ }^{5}$ Although these findings gave silicone implants promise for the future advancement of breast reconstructive surgery, inaccurate and biased media reporting have created negative perceptions regarding their use. ${ }^{6}$ Sigurdson and Lalonde reported that a systematic review of 24 studies by a US court-ordered national science panel concluded that there is no association between silicone implants and connective tissue disease. ${ }^{6}$ However, these studies notwithstanding, recent studies strongly suggest an increase in acute large cell lymphoma with prosthetic implants.

Breast reconstruction has improved dramatically over the last century as a surgical solution to anatomical deformities caused by breast cancer. Although success has been seen in the surgical technique, as well as improvement in self-image, breast reconstruction does not impede standard oncologic treatment, does not delay detection of recurrent cancer, and does not change the overall mortality associated with the disease. ${ }^{1,4}$ However, breast reconstruction does not come without associated risks. As these complications are studied further and surgical techniques and implants improved, these efforts will undoubtedly assist with the enhancement of breast reconstruction surgery as we move forward.

\section{Disparities and psychologic effects of mastectomy}

Breast reconstruction after mastectomy is still not routinely being performed on all patients because many patients are unaware and/or uninformed of their options preoperatively.
At times, due to the overwhelming nature of the recent diagnosis, after a detailed discussion with their surgeons with regard to risks and benefits, some patients feel compelled to make decisions without full comprehension. ${ }^{7}$ Patients requesting reconstruction to maintain their body image should be made aware that the reconstructed breast will not look exactly like their natural breast. ${ }^{6}$ Faced with such knowledge, the patient should make a decision whether or not to pursue reconstruction, and specifically which type of surgery she would prefer. ${ }^{7}$

The decision to get immediate breast reconstruction versus delayed reconstruction or avoiding reconstruction altogether has been related to patient demographics. Reuben et al demonstrated that immediate reconstruction was more likely to happen in the younger (less than 50 years) white patient who was seeking medical care in an urban versus a rural hospital. ${ }^{8}$ These patients were also more likely to be educated, employed, and married. ${ }^{7,8}$ However, recently there has been an increase in breast reconstruction among older patients (greater than 65 years), probably related to greater awareness and changes in provider bias. ${ }^{8}$ Patients who did not undergo immediate reconstruction were more likely to be older, with multiple comorbidities and receiving care in a nonteaching hospital. ${ }^{8}$ With regard to race, patients who were African American were more unlikely to get immediate reconstruction in comparison with White, Hispanic, and Native American patients.

Patient insurance has had a role to play in whether or not a patient would get immediate breast reconstruction. Patients with private/health maintenance organization insurance were more likely to get immediate reconstruction in comparison with patients having government-sponsored insurance, such as Medicare and Medicaid. ${ }^{7,8}$

However, Panieri et al studied a smaller group and found quite the opposite, with no association between immediate reconstruction, age, occupation, education level, and marital status. Patients in this study preferred to have simpler procedures, and expressed less concern for their postoperative appearance. ${ }^{7,9}$

Breast reconstruction arose from the perceived emotional distress triggered by mastectomy, thus there have been studies attempting to elucidate exactly what impact reconstruction has on body image, sexuality, and quality of life after surgery.

Al-Ghazal et al reported that patients having immediate breast reconstruction had improved overall satisfaction, better body image, better self-esteem, and feelings of sexual attractiveness. ${ }^{7,10}$ There was less depression and 
anxiety compared with patients who underwent delayed reconstruction. Knottenbelt et al also reported a decrease in stress post mastectomy by restoring body image. ${ }^{7,11}$

Harcourt et al performed a prospective study involving 103 patients undergoing mastectomy with or without reconstruction. The patients were assessed preoperatively, and six and 12 months postoperatively with regards to psychological distress, quality of life, and body image. ${ }^{3}$ One year postoperatively, there was increased evidence of depression among all except those who had delayed reconstruction. ${ }^{3}$ Throughout the study, patients assessed their body image and at the end of one year, poor body image was reported by those who had mastectomy and those who had mastectomy with immediate reconstruction, which was $36 \%$ and $29 \%$, respectively. ${ }^{3}$ It was found that patients had a poorer body image at six months and one year after surgery if they were at a younger age at the start of the study and if there was evidence of depressive symptoms. ${ }^{3}$ At the end of the study period, the women who had delayed reconstruction were the most satisfied group, which is the opposite of what has been seen in prior studies where the highest level of satisfaction was among the group of patients who had immediate reconstruction. ${ }^{3}$

\section{Breast reconstruction Autologous}

Reconstruction can be done as a one-stage procedure immediately following mastectomy or in a delayed fashion in which reconstruction is done weeks to months later. In general, autologous reconstruction as compared with prosthetic reconstruction, provides a more natural-feeling breast and avoids the use of a foreign body. ${ }^{6,12}$ Research is advancing quickly and developing a myriad of approaches and techniques to reconstruct breasts that are more esthetically pleasing and associated with fewer complications and donor site morbidities. Microsurgical breast reconstruction is a modality that involves the use of remote soft tissues and their integral neurovasculature to reconstruct the distant mastectomy site. ${ }^{13}$ Microsurgery is indicated in several situations, including patients with previous or anticipated chest wall radiation treatment. It is also an ideal option for patients with a ptotic contralateral breast and previous failed implant reconstruction. ${ }^{1}$ Furthermore, many autologous reconstruction operations may be performed without modification of the contralateral breast to restore symmetry and contour, as is often difficult to achieve with the use of implants. ${ }^{6}$

The most popular autologous reconstruction utilizes the TRAM flap (pedicled or free). The TRAM flap is most useful in patients with small-sized to normal-sized breasts and those who may have breast ptosis. , $^{12}$ This flap may not be ideal for patients with larger breasts because of the risk of flap necrosis should the volume of the flap exceed available blood flow. ${ }^{6}$ Patients who require bilateral reconstruction are not ideal candidates for the TRAM flap because of a significant donor site defect as well decreased abdominal strength which may later require abdominal wall reconstruction. ${ }^{6,12}$

Microsurgical breast reconstruction has evolved over the past 30 years as surgical innovation is advancing. Given that the use of autologous tissues for reconstruction has become the gold standard, microsurgical procedures have become more widely used. The many different procedures that have been used for microsurgical breast reconstruction include, but are not limited to, the free TRAM flap, the SIEA flap, the DIEP flap, and the thoracodorsal artery perforator flap. ${ }^{1,6}$

The free TRAM flap is harvested as a free flap and by using the more dominant arterial supply and venous drainage of the lower abdomen, allowing the harvesting of larger volumes of tissue without increasing the risk for the development of fat necrosis. ${ }^{6}$ Complications associated with free TRAM flaps include microvascular thrombosis resulting in flap loss and longer operation time. ${ }^{4,6}$ Sigurdson and Lalonde reported flap loss in $0.3 \%-0.9 \%$ of free TRAM flap cases in high-volume centers. ${ }^{6}$

Another use of the deep inferior epigastric vascular system involves the free DIEP flap. ${ }^{1,6}$ Its advantages include less rectus muscle nerve damage, lower rates of abdominal wall herniation and bulge formation, less pain, and shorter hospital stay. ${ }^{6}$ As a result of shorter hospital stay, free DIEP flaps have been found to be more cost-effective than TRAM flaps. ${ }^{6}$ The free SIEA flap does not violate the abdominal wall fascia and is capable of supporting enough abdominal tissue volume to create an adequate breast mound. ${ }^{1}$ Sigurdson and Lalonde reported that these vessels are only suitable for use in $30 \%$ of patients, and are generally indicated for women with A or B cup breast sizes because the vascular territory of the flap is limited based on vessel size. ${ }^{6}$

The latissimus dorsi musculocutaneous flap can be used with or without implants and is an option for replacing radiationdamaged skin. If desired, an implant can be inserted posterior to the muscle, although high capsular contraction rates $(21 \%-75 \%)$ have been reported. ${ }^{12}$ In selected patients, the flap has proven to be another good option for reconstruction, particularly in those with previous abdominal surgery, failed previous reconstruction, or as an adjunct in combination with an implant for better skin coverage. ${ }^{1,14}$ In addition to the complications associated with implant-based reconstruction (delayed healing, capsular contracture, implant malposition, implant extrusion, infection), 
the latissimus dorsi musculocutaneous flap carries the risk of significant donor site morbidity. ${ }^{6}$ Other possible complications include contour irregularity of the back, seroma formation (47\%-96\%), and shoulder weakness. ${ }^{1,6}$

Plastic surgeons are continuously advancing their search for additional, more dynamic, operative techniques, in spite of the wide variety of options already available. The thoracodorsal artery perforator flap uses an identical vascular pedicle and tissues to those of the latissimus flap, and serves as a potential solution for large quadrantectomy defects. ${ }^{6}$ Other free perforator flaps, such as the superior gluteal artery perforator flap, the inferior gluteal artery perforator flap, and the transverse upper gracilis flap are also being studied. ${ }^{6}$

Although microsurgery offers significant advancement in the evolution of breast reconstruction, the procedures are far from perfect. Like any surgery, there are complications and risks that even the most experienced of surgeons may not be able to avoid. The flaps used in these procedures are not immune to fat necrosis, fibrosis, atrophy, or contracture. Outcomes vary with immediate versus delayed approaches. ${ }^{4}$ Research is steadfastly aimed at minimizing any unfavorable outcomes. Despite these shortcomings, breast reconstruction maintains excellent potential to restore anatomic contour and symmetry, thus improving quality of life.

\section{Prosthetic}

Prosthetic breast reconstruction is the most frequently performed type of breast reconstruction in the US and Canada. ${ }^{15,16}$ Because of its supreme biocompatibility as well as elastic and deformable properties, silicone has emerged as the material of choice. All prosthetic devices are hollow shells fabricated from a relatively tough silicone capsule filled with either saline or silicone gel. ${ }^{6,16}$ Modern permanent implants come in a variety of sizes. They can be either smooth or textured, with some of the saline prostheses having the capability of postoperative size adjustment. ${ }^{17-19}$ Prosthetic breast reconstruction is generally meant to be a staged process. ${ }^{18}$ Initially the expander device is implanted post mastectomy either immediately or in delayed chronology. Once the tissues have been sufficiently expanded, the temporary device is removed and replaced with the permanent implant of choice. ${ }^{12,16}$ In some cases, the contralateral breast has such favorable morphology that immediate insertion of a permanent implant allows reasonably good simulation in one stage.

\section{Adjuvant therapy}

Depending on the clinical and pathologic stage of the malignancy, the adjunctive use of chemotherapy and/or radiation therapy is not uncommon. Whether performing prosthetic or autologous reconstruction, the effects of chemotherapy can be mitigated by appropriate timing of surgical interventions. Chemotherapy is usually given 4-6 weeks after surgery and potentially can be delayed if there are wound complications from reconstruction. ${ }^{7}$ Wilson et al compared patients undergoing immediate reconstruction following breast conservation therapy or mastectomy, and found no difference between the two groups with respect to initiation of chemotherapy. ${ }^{20}$ Taylor and Kumar found a delay of approximately five days after TRAM flap due to issues with the wound. ${ }^{21}$ Mortenson et al found an increase in wound complications in patients who underwent immediate reconstruction compared with those who did not. ${ }^{22}$

Radiotherapy following breast reconstruction has been associated with adverse cosmetic outcomes. ${ }^{7}$ The effects of radiation therapy tend to be more difficult to negotiate in prosthetic reconstruction. ${ }^{4,12,23}$ Capsular contractures, distortion of the reconstructed breast, and chronic chest pain are a few complications occurring at rates as high as $50 \%$ after implant reconstruction. ${ }^{4,7,23}$ The effects of radiation vary by dosage, duration, type, and from person to person. Radiation severely impairs wound healing and can cause irreversible damage to involved tissues, with effects seen up to several years later., ${ }^{4,24}$ Multiple salvaging techniques are available to address complications of radiation. Capsulotomy and capsulectomy are often the first line of defense. Interposition or major supplementation of the treatment area with regional pedicled flaps is often necessary to rectify extensive problems. With careful case selection and proper preparation, prosthetic reconstruction in the face of radiation can be successful and satisfying.

Recent studies have suggested that while autologous tissue is better able to withstand radiation, it is also susceptible to its damaging effects. The rate of fat necrosis, flap volume loss, and contracture is still significant, particularly in immediate reconstruction. ${ }^{4,7}$ Choi et al emphasize the challenge of identifying patients who require post mastectomy radiation prior to mastectomy and reconstruction. ${ }^{4}$ Kronowitz et al addressed this by utilizing a delayed-immediate reconstructive strategy in which patients remained in hospital following skin-sparing mastectomy with expander placement. ${ }^{25}$

\section{The contralateral breast}

Depending on the anatomic variations conferred by age and a myriad of environmental factors, the contralateral breast can be made more anatomic by either reduction, mastopexy, or in some cases augmentation. ${ }^{26,27}$ This procedure can 
be performed at the same time as the mastectomy. ${ }^{17}$ This restoration of a more ideal contralateral breast form will offer a reasonable index and endpoint to be approached in reconstructing the oncologically treated breast. With proper timing and planning, such procedures will not necessarily extend the duration of reconstruction, and will only enhance the final outcome and patient satisfaction.

In 2010, Leone et al studied at least 600 patients who had unilateral mastectomy and reconstruction over a seven-year period. ${ }^{28}$ Procedures included mastopexy, augmentation, and reduction mastopexy. Patients who had delayed reconstruction were more likely to require a symmetrization when compared with those undergoing immediate reconstruction. ${ }^{28}$ They also found the percentage of contralateral procedures was higher for patients undergoing reconstruction with implants and in patients who had a traditional versus a skin-sparing mastectomy. Skin-sparing mastectomy with immediate implant breast reconstruction resulted in fewer symmetrization procedures and the best cosmetic outcome. ${ }^{28}$

In addition to creating symmetry, there is also the issue of creation of a nipple for patients who undergo a traditional mastectomy. For patients undergoing reconstruction with implants, the creation of a nipple can be performed after the expander is removed and replaced with a silicone implant. Of all the historic forms of nipple areolar reconstruction, the current standard of care has come down to intrinsic random nipple flaps with concurrent or delayed tattooing. Even with nipple-sparing mastectomy, there is often the need to support or repigment a flaccid pale nipple-areola complex.

\section{The future}

Skin-sparing mastectomy continues to grow in popularity and has approached standard of care status with breast reconstruction. This technique maintains the breast skin envelope as well as the inframammary folds while removing all the glandular tissue of the breast and the nipple-areola complex. ${ }^{4,29}$ The inframammary fold, intermammary crease, and a well formed breast envelope are cornerstones to an anatomic and natural appearing reconstruction. ${ }^{4,29}$ If maintained, these structures greatly enhance reconstruction with either an implant or autologous tissues. ${ }^{4}$ Breast envelope preservation makes recreation of the breast less arduous, with fewer repeat visits to the operating room in order to achieve a good anatomic outcome. Even though skin-sparing mastectomy is esthetically more pleasing, there are concerns about the risk of local recurrence. Carlson et al concluded from a large retrospective series that the risk of recurrence post skin-sparing mastectomy is related to tumor stage, grade, and lymphovascular proliferation., ${ }^{7,30}$ There have been several retrospective studies, including a 15 -year study by Greenway et al, comparing skin-sparing mastectomy with conventional mastectomy. ${ }^{31}$ For early-stage breast cancer (stage I and II) they have shown that the risk of local recurrence is comparable with that after conventional mastectomy. The safety of skin-sparing mastectomy has been shown for early-stage cancers, but for stage III and more advanced cancers, studies have shown that there is an increased risk of local recurrence. ${ }^{31}$ Medina-Franco et al evaluated 270 patients over a median period of 70 months and noted that patients with stage III disease had a local recurrence rate of $31 \%$ compared with $5.8 \%$ in the earlier stages. The patient satisfaction rate with postoperative reconstruction has been quoted to be as high as $90 \% .^{32}$

Nipple-sparing mastectomy is that in which the nippleareola complex is left in situ, with dissection of the deep glandular tissue. ${ }^{29}$ This approach to mastectomy has the advantage of eliminating the need for nipple reconstruction at a later date, with maintenance of the breast skin envelope to give the natural contour and texture of the patient's breast. ${ }^{29}$ There is a risk of local recurrence for multicentric tumors as well as subareola tumors. To decrease the risk of local recurrence, surgeons have been doing frozen sections of the retro-areola tissue intraoperatively. There is a significant concern for the risk of local recurrence as well as loss of the nipple-areola complex. ${ }^{33}$

Sacchini et al in 2006 studied 123 patients who underwent nipple-sparing mastectomy with immediate reconstruction and found that even though there were recurrences in three patients, there was no recurrence in the nipple-areola complex, 22 patients (11\%) developed nipple necrosis, and $60 \%$ of them thought that it was minimal, and, overall, the patients reported satisfaction with the cosmetic outcome. ${ }^{33}$ The risk of nipple necrosis is based on the incision used for the mastectomy and its proximity to the nipple, as documented by Wijayanayagam et al. ${ }^{34}$

Areola-sparing mastectomy involves removal of the nipple and requires reconstruction of the nipple at a later date. Simmons et al noted that the areola was involved in only two of 23 patients with involvement of the nipple-areola complex and supports this approach. They further studied a group of 17 cases over a 20 -month period and found wound infection to be the sole complication. ${ }^{35}$ However, at this time, larger studies are needed before any conclusions can be made.

Even though many women are treated with mastectomy for breast cancer, at this time breast conservation therapy is also considered standard of care. As discussed earlier 
regarding the reconstructive techniques available for a patient undergoing mastectomy, we must also consider techniques to optimize a patient's body image after breast conservation therapy. This relatively new field has been termed "oncoplastic" surgery and involves reconstruction, either immediate or delayed, post breast conservation surgery. ${ }^{36}$ This area was developed due to the poor esthetic outcome noted in up to $20 \%-30 \%$ of patients after breast conservation therapy, especially when large volumes of breast tissue are removed and with the additive effects of radiotherapy on cosmesis. ${ }^{36}$ The great concern is whether or not the procedure is oncologically safe as well as predictive of a good cosmetic outcome. Fitoussi et al studied 540 patients over a 12 -year period and monitored overall survival, disease-free survival, and rates of local recurrence, as well as grades for the esthetic outcome. At five-years, the rate of overall survival was $93 \%$, disease-free survival was $88 \%$, and local recurrence was $6.8 \%$. Analyzing the esthetic outcome, $98 \%$ of patients had a good outcome at one year, and $7.8 \%$ of patients required revisional surgery due to cosmetic issues. ${ }^{36}$

This shows that oncoplastic surgery has good results in terms of survival and esthetic outcome, and should be considered in all patients who are good candidates for breast conservation surgery. For those candidates not suitable for breast conservation therapy, consideration should be given to skin-sparing mastectomy with immediate reconstruction, given the superior results.

\section{Disclosure}

The authors report no conflicts of interest in this work.

\section{References}

1. Losee JE, Gimbel M. Plastic and Reconstructive Surgery: Schwartz's Principles of Surgery. New York, NY: McGraw Hill; 2010.

2. Dunnington G, Andriole D, Kaiser S. Breast: Essentials of General Surgery. Philadelphia, PA: Lippincott; 2006.

3. Harcourt DM, Ramsey NJ, Ambler NR, et al. The psychological effect of mastectomy with or without breast reconstruction: A prospective, multi-center study. Plast Reconstr Surg. 2003;111:1060-1068.

4. Choi JY, Alderman AK, Newman LA. Aesthetic and reconstructive considerations in oncologic breast surgery. J Am Coll Surg. 2006;202: 943-944.

5. Bondurant S, Ernster V, Herdman R, editors. Institute of Medicine (US) Committee on the Safety of Silicone Breast Implants. Safety of Silicone Breast Implants. Washington, DC: National Academies Press; 1999.

6. Sigurdson L, Lalonde D. MOC-PSSM CME article: Breast reconstruction. Plast Reconstr Surg. 2008;121:1-12.

7. Fernández-Frías AM, Aguilar J, Sánchez JA, Merck B, Piñero A, Calpena R. Immediate reconstruction after mastectomy for breast cancer: Which factors affect its course and final outcome? JAm Coll Surg. 2009; 208:126-133.
8. Reuben BC, Manwaring J, Neumayer LA. Recent trends and predictors in immediate breast reconstruction after mastectomy in the United States. Am J Surg. 2009; 198:237-243.

9. Panieri E, Lazarus D, Dent DM, Hudson DA, Murray E, Werner ID. A study of the patient factors affecting reconstruction after mastectomy for breast carcinoma. Am Surg. 2003;69:95-97.

10. Al-Ghazal SK, Sully L, Fallowfield L, et al. The psychological impact of immediate rather than delayed breast reconstruction. Eur J Surg Oncol. 2000;26:17-19.

11. Knottenbelt A, Spauwen PHM, Wobbes TH. The oncological implications of immediate breast reconstruction. Eur J Surg Oncol. 2004;30:829-833.

12. Abdalla HM, Shalaan MA, Fouad FA, Elsayed AA. Immediate breast reconstruction with expander assisted latissimus dorsi flap after skin sparing mastectomy. J Egypt Natl Canc Inst. 2006;18:134-140.

13. Avraham T, Clavin N, Mehrara B. Microsurgical breast reconstruction. Cancer J. 2008;14:241-247.

14. Townsend CM Jr, Beauchamp RD, Evers BM, Mattox KL, editors. Sabiston Textbook Of Surgery: The Biological Basis of Modern Surgical Practice. 18th ed. Philadelphia, PA: WB Saunders; 2008.

15. Spear SL, Mardini S, Ganz JC. Resource cost comparison of implantbased breast reconstruction versus TRAM flap breast reconstruction. Plast Reconstr Surg. 2003;112:101-105.

16. Sbitany H, Sandeen SN, Amalfi AN, Davenport MS, Langstein HN. Acellular dermis-assisted prosthetic breast reconstruction versus complete submuscular coverage: A head-to-head comparison of outcomes. Plast Reconstr Surg. 2009;124:1735-1740.

17. Shestak KC, Szem JW, Zabel DD, Nastala CL. Breast reconstruction. In: Weinzweig J, editor. Plastic Surgery Secrets. Philadelphia, PA: Hanley and Belfus; 1999.

18. Beasley ME. Two-stage expander/implant reconstruction: Delayed. In: Spear SL, editor. Surgery of the Breast: Principles and Art. Philadelphia, PA: Lippincott-Raven; 1998.

19. Spear SL. Prosthetic reconstruction in the radiated breast. In: Spear SL, editor. Surgery of the Breast: Principles and Art. 2nd ed. Philadelphia, PA: Lippincott-Raven; 2005.

20. Wilson CR, Brown IM, Weiller-Mithoff E, et al. Immediate breast reconstruction does not lead to a delay in the delivery of adjuvant chemotherapy. Eur J Surg Oncol. 2004;30:624-627.

21. Taylor CW, Kumar S. The effect of immediate breast reconstruction on adjuvant chemotherapy. Breast. 2005;4:18-21.

22. Mortenson MM, Schneider PD, Khatri VP, et al. Immediate breast reconstruction after mastectomy increases wound complications. Arch Surg. 2004;139:988-991.

23. Spear S, Onyewu C. Staged breast reconstruction with saline-filled implants in the irradiated breast: Recent trends and therapeutic implications. Plastic Reconstr Surg. 2000;105:930-942.

24. Tran NV, Evans GR, Kroll SS, et al. Postoperative adjuvant irradiation: Effects on transverse rectus abdominis muscle flap. Plast Reconstr Surg. 2000;106:313-317.

25. Kronowitz SJ, Hunt KK, Kuerer HM, et al. Delayed-immediate breast reconstruction. Plast Reconstr Surg. 2004;113:1617-1628.

26. Nahabedian M. Breast reconstruction: A review and rationale for patient selection. Plast Reconstr Surg. 2009;124:55-62.

27. Munhoz AM, Montag E, Arruda EG, et al. Critical analysis of reduction mammaplasty techniques in combination with conservative breast surgery for early breast cancer treatment. Plast Reconstr Surg. 2006; 117:1091-1103.

28. Leone MS, Priano V, Franchelli S, et al. Factors affecting symmetrization of the contralateral breast: A 7-year unilateral postmastectomy breast reconstruction experience. Aesthetic Plast Surg. December 7, 2010. [Epub ahead of print].

29. Reefy S, Patani N, Anderson A, Burgoyne G, Osman H, Mokbel K. Oncological outcome and patient satisfaction with skin-sparing mastectomy and immediate breast reconstruction: A prospective observational study. BMC Cancer. 2010;10:171. 
30. Carlson GW, Styblo TM, Lyles RH, et al. Local recurrence after skinsparing mastectomy: Tumor biology or surgical conservatism? Ann Surg Oncol. 2003;10:108-112.

31. Greenway RM, Schlossberg L, Dooley WC. Fifteen-year series of skin sparing mastectomy for stage 0 to 2 breast cancer. Am J Surg. 2005;190: 918-922.

32. Medina-Franco H, Vasconez LO, Fix RJ, et al. Factors associated with local recurrence after skin-sparing mastectomy and immediate breast reconstruction for invasive breast cancer. Ann Surg. 2002;235: 814-819.

33. Sacchini V, Pinotti JA, Barros AC, et al. Nipple-sparing mastectomy for breast cancer and risk reduction: Oncologic or technical problem. J Am Coll Surg. 2006;203:704-714.
34. Wijayanayagam A, Kumar AS, Foster RD, Esserman LJ. Optimising the total skin sparing mastectomy. Arch Surg. 2008;143:38-45.

35. Simmons RM, Brennan M, Christos P, King V, Osborne M. Analysis of nipple/areolar involvement with mastectomy: Can the areola be preserved? Ann Surg Oncol. 2002;9:165-168.

36. Fitoussi AD, Berry MG, Fama F, et al. Oncoplastic breast surgery for cancer: Analysis of 540 consecutive cases. Plast Reconstr Surg. 2010; $125: 2: 454-462$.

\section{Publish your work in this journal}

Breast Cancer: Targets and Therapy is an international, peerreviewed open access journal focusing on breast cancer research, identification of therapeutic targets and the optimal use of preventative and integrated treatment interventions to achieve improved outcomes, enhanced survival and quality of life for the cancer patient.

\section{Dovepress}

View the full aims and scopes of this journal here. The manuscript management system is completely online and includes a very quick and fair peer-review system, which is all easy to use. Visit http:// www.dovepress.com/testimonials.php to read real quotes from published authors.

Submit your manuscript here: http://www.dovepress.com/breast-cancer---targets-and-therapy-journal 